\title{
Interaction of multidimensional convection and radial pulsation
}

\author{
Chris M. Geroux ${ }^{1}$ and Robert G. Deupree ${ }^{2}$ \\ ${ }^{1}$ Institute for Computational Astrophysics and Department of Astronomy and Physics, \\ Saint Mary's University, Halifax, NS B3H 3C3 Canada. \\ ${ }^{2}$ Now at Physics and Astronomy, University of Exeter, Stocker Road, Exeter, UK EX4 4QL \\ email: geroux@astro.ex.ac.uk
}

\begin{abstract}
We have previously calculated a number of 2D hydrodynamic simulations of convection and pulsation to full amplitude. These revealed a significantly better fit to the observed light curves near the red edge of the instability strip in the globular cluster M 3 than did previous 1D mixing length models. Here we compare those 2D results with our new 3D hydrodynamic simulations calculated with the same code. As expected, the horizontal spatial behaviour of convection in $2 \mathrm{D}$ and $3 \mathrm{D}$ is quite different, but the time dependence of the convective flux on pulsation phase is quite similar. The difference in pulsation growth rate is only about $0.1 \%$ per period, with the 3D models having more damping at each of the five effective temperatures considered. Full amplitude pulsation light curves in 2D and 3D are compared.
\end{abstract}

Keywords. convection, hydrodynamics, methods: numerical, stars: oscillations, stars: variables

\section{Introduction}

The importance of RR Lyrae and classical Cepheids as standard candles has led to a long history of trying to model the pulsation of these variables (e.g. Christy 1964, Bono \& Stellingwerf 1994) with one-dimensional (1D) hydrodynamic simulations. It was speculated early on that convection in the ionization regions for models near the red edge would be important (Christy 1966, Cox et al. 1966).

We present an alternative to $1 \mathrm{D}$ time-dependent mixing length based approaches to convection in pulsation models (e.g. Stellingwerf 1982a,b; Kuhfuss 1986; Xiong 1989) with the aim to improve agreement with observations, specifically near the red edge of the RR Lyrae instability strip where the treatment of convection is especially important. Our approach (similar to Deupree 1977) is to follow the convective flow directly using the normal conservation laws of hydrodynamics in both $2 \mathrm{D}$ and $3 \mathrm{D}$. Recently others have begun to perform 2D calculations of this nature (e.g. Mundprecht et al. 2013, Gastine \& Dintrans 2011) but these have not yet been compared with observations. Our multidimensional full amplitude radial pulsation calculations have been made possible by using a moving grid system that adjusts the volume of the radial shells to keep the total mass in the shell constant (see Geroux \& Deupree 2011, 2013 for details).

\section{Key results}

2D models compared with observations. We have compared our model light curves to light curves of RR Lyrae variables in M 3 as observed by Corwin \& Carney (2001), finding reasonable agreement between our fundamental-mode model light curves from the near the fundamental blue edge $\left(T_{\text {eff }}=6700 \mathrm{~K}\right)$ to near the fundamental red edge $\left(T_{\text {eff }}=6300 \mathrm{~K}\right)$. Our single first-overtone model light curve also agrees reasonably well 
with observed light curves. A comparison of the modelled visual amplitude - effective temperature relation with that derived form data from Corwin \& Carney shows good agreement for both our fundamental-mode models and our single first-overtone model.

$3 D$ convective flow patterns. The up-flow filling factor, $f_{\mathrm{up}}$ (fraction of the area with positive radial velocities) is phase dependent. When the model has expanded $f_{\text {up }} \approx 0.7$ and when contracted $f_{\text {up }} \approx 0.4$. The time average of the filling factor, $\left\langle f_{u p}\right\rangle$, is about 0.6 . The values of $\left\langle f_{\text {up }}\right\rangle$ and $f_{\text {up }}$ during contraction and expansion are quite similar for both the $6300 \mathrm{~K}$ and $6700 \mathrm{~K}$ models with the value of $\left\langle f_{\text {up }}\right\rangle$ reasonably close to that found by other authors of about 2/3 (Magic et al. 2013, Stein \& Nordlund 1998). Simplistic measurements of granule sizes produce $\log d_{\text {gran }}=10.5$ and 10.3 for the $6300 \mathrm{~K}$ and $6700 \mathrm{~K}$ effective-temperature models respectively. This agrees with relations from Magic et al. (2013), derived from high resolution atmosphere models which gives $\log d_{\text {gran }}=$ 10.2 , and 10.18 for the $6300 \mathrm{~K}$ and $6700 \mathrm{~K}$ models respectively. This order of magnitude agreement suggests that our coarsely horizontally zoned calculations $(20 \times 20)$ are likely obtaining approximately the correct granule sizes and filling factors for the largest eddies. However, due to the coarse horizontal zoning we cannot hope to resolve smaller scale features.

Comparison of $2 D$ and $3 D$ models. We find good agreement both in terms of shape and amplitude between the 2D and $3 \mathrm{D}$ light curves of $6300 \mathrm{~K}$ and $6600 \mathrm{~K}$ models. The hotter model does, however, have some differences in shape during the descending light. The difference in pulsational growth rate between $2 \mathrm{D}$ and $3 \mathrm{D}$ models is only about $0.1 \%$ with 3D models having more damping.

Time dependence of $2 D$ and $3 D$ convection. The time dependence we found was very similar to that found by Deupree (1977) which led to the quenching of pulsation near the red edge, namely that the convective flux is a maximum during full contraction, and a minimum during full expansion. While the 2D and 3D calculations show the same time dependence, there are some differences. Most notably the 3D maximum convective fluxes are larger due to larger down flow velocities and slightly larger horizontal temperature variations. The total convective luminosity of the $3 \mathrm{D}$ calculations is actually slightly smaller. The reason this is true, while having larger maximum convective fluxes, is that the filling factors of the strong down-flows are smaller in $3 \mathrm{D}$ than $2 \mathrm{D}$.

\section{References}

Bono, G. \& Stellingwerf, R. F. 1994, ApJS, 93, 233

Christy, R. F. 1964, Reviews of Modern Physics, 36, 555

Christy, R. F. 1966, ApJ, 144, 108

Corwin, T. M. \& Carney, B. W. 2001, AJ, 122, 3183

Cox, J. P., Cox, A. N., Olsen, K. H., King, D. S., \& Eilers, D. D. 1966, ApJ, 144, 1038

Deupree, R. G. 1977, ApJ, 211, 509

Gastine, T. \& Dintrans, B. 2011, $A \& A$, 528, A6

Geroux, C. M. \& Deupree, R. G. 2011, ApJ, 731, 18

Geroux, C. M. \& Deupree, R. G. 2013, ApJ, 771, 113

Kuhfuss, R. 1986, A\& A, 160, 116

Magic, Z., Collet, R., Asplund, M., et al. 2013, A\&SA, 557, A26

Mundprecht, E., Muthsam, H. J., \& Kupka, F. 2013, MNRAS, 435, 3191

Stein, R. F. \& Nordlund, Å. 1998, ApJ, 499, 914

Stellingwerf, R. F. 1982a, ApJ, 262, 330

Stellingwerf, R. F. 1982b, ApJ, 262, 339

Xiong, D. 1989, A\& A, 209, 126 